\title{
Influence of anticoagulants on the risk of delayed bleeding after gastric endoscopic submucosal dissection: a multicenter retrospective study
}

\author{
Hideomi Tomida ${ }^{1,2} \cdot$ Toshiyuki Yoshio $^{3}$ (D) $\cdot$ Kimihiro Igarashi $^{4} \cdot$ Yoshinori Morita $^{5} \cdot$ Ichiro Oda $^{6} \cdot$ Takuya Inoue $^{7}$. \\ Takuto Hikichi $^{8}$. Tetsuya Sumiyoshi $^{9} \cdot$ Hisashi Doyama $^{10}$. Yosuke Tsuji ${ }^{11}$. Jun Nishikawa ${ }^{12}$. Waku Hatta ${ }^{13}$. \\ Tatsuya Mikami ${ }^{14} \cdot$ Mikitaka Iguchi $^{15} \cdot$ Kazuki Sumiyama $^{16} \cdot$ Katsumi Yamamoto $^{17} \cdot$ Kazuya Kitamura $^{18}$. \\ Shiko Kuribayashi ${ }^{19}$. Atsushi Yanagitani ${ }^{20}$. Toshio Uraoka $^{21} \cdot$ Tomoyuki Yada $^{22} \cdot$ Kenkei Hasatani $^{23}$. \\ Koichiro Kawaguchi ${ }^{24} \cdot$ Tomoki Fujita $^{25} \cdot$ Tsutomu Nishida $^{26} \cdot$ Yoichi Hiasa $^{1} \cdot$ Mitsuhiro Fujishiro $^{27} \cdot$ for the FIGHT- \\ Japan Study Group
}

Received: 3 June 2020 / Accepted: 9 July 2020 / Published online: 18 July 2020

(c) The International Gastric Cancer Association and The Japanese Gastric Cancer Association 2020

\begin{abstract}
Background and aims Delayed bleeding after gastric endoscopic submucosal dissection (ESD) in patients receiving anticoagulants remains an unpreventable adverse event. Although direct-acting oral anticoagulants (DOACs) have superior efficacy in preventing thromboembolism, their effects on the occurrence of delayed bleeding remain unclear. This study aimed to elucidate the clinical effect of DOACs on delayed bleeding after gastric ESD.

Patients and methods We retrospectively examined 728 patients who received anticoagulants and were treated for gastric neoplasms with ESD in 25 institutions across Japan. Overall, 261 patients received DOACs, including dabigatran (92), rivaroxaban (103), apixaban (45) and edoxaban (21), whereas 467 patients were treated with warfarin.

Results Delayed bleeding occurred in $14 \%$ of patients taking DOACs, which was not considerably different in patients receiving warfarin (18\%). Delayed bleeding rate was significantly lower in patients receiving dabigatran than in those receiving warfarin and lower than that observed for other DOACs. Multivariate analysis showed that age $\geq 65$, receiving multiple antithrombotic agents, resection of multiple lesions and lesion size $\geq 30 \mathrm{~mm}$ were independent risk factors, and that discontinuation of anticoagulants was associated with a decreased risk of bleeding. In multivariate analysis among patients taking DOACs, dabigatran therapy was associated with a significantly lower risk of delayed bleeding.

Conclusions The effects of DOACs on delayed bleeding varied between agents, but dabigatran therapy was associated with the lowest risk of delayed bleeding. Switching oral anticoagulants to dabigatran during the perioperative period could be a reasonable option to reduce the risk of delayed bleeding after gastric ESD.
\end{abstract}

Keywords Gastric endoscopic submucosal dissection $\cdot$ Delayed bleeding $\cdot$ Direct-acting oral anticoagulants $\cdot$ Dabigatran

Electronic supplementary material The online version of this article (https://doi.org/10.1007/s10120-020-01105-0) contains supplementary material, which is available to authorized users.

Toshiyuki Yoshio

toshiyuki.yoshio@jfcr.or.jp

Extended author information available on the last page of the article

\section{Introduction}

Endoscopic submucosal dissection (ESD) is a globally performed, minimally invasive treatment for node-negative, early gastric cancer (EGC) [1] with relatively favorable long-term outcomes, comparable to those of surgical treatment [2]. However, post-ESD delayed bleeding occurs as an uncontrolled adverse event, in $3.1 \%-6.5 \%$ of cases [3, 4], a rate which has not decreased over the past decade.

In the aging society, the number of patients taking antithrombotic agents may be expected to rise as a preventative therapy for cardio- and cerebrovascular diseases. 
Accordingly, we are observing an increasing number of patients undergoing ESD within the context of ongoing treatments with antithrombotic agents including anticoagulants and antiplatelet agents. Antithrombotic agents may increase the risk of delayed bleeding, depending on their type and dose, and on whether they are discontinued or continued during the perioperative period [5-9]. Anticoagulants, which include warfarin and direct-acting oral anticoagulants (DOACs), are difficult to manage during the perioperative period of gastric ESD because anticoagulant therapies represent most potent risk factors of delayed bleeding $[7,9]$ and of secondary thrombotic events $[7,10,11]$. In the past, heparin-bridging therapy (HBT) together with discontinuation of warfarin therapy was used as an approach to prevent thrombotic surgery-related events [12]. However, HBT caused a remarkable risk of delayed bleeding following surgical and interventional procedures [13] as well as gastric ESD [7, 9, 14]. Recent studies further revealed that HBT does not reduce perioperative arterial thromboembolism [15-17]. These findings underscore the need for new strategies to manage warfarin during the perioperative period. While the continuation of warfarin therapy and switching warfarin to DOACs are candidate approaches, data supporting their use are still lacking [10].

Several studies demonstrated an increased risk of gastrointestinal bleeding among DOACs-treated patients [18-20]. However, these results do not apply to patients receiving endoscopic treatment, and those who may have post-procedural wounds in specific organs rather than spontaneous bleeding. Moreover, these studies assessed effects on patients treated with warfarin and not on those receiving individual DOACs.

We previously reported that patients receiving dabigatran had a lower risk of delayed bleeding after gastric ESD than those receiving rivaroxaban [9]. However, this study was not adequately powered to establish the comparative clinical efficacy of different DOACs, due to the small number of patients being treated with DOACs (particularly with apixaban and edoxaban). Several studies have been conducted to determine the effect of anticoagulants on endoscopic procedures; DOACs has been more favorable in bleeding complications than warfarin [21], and high-risk procedures have been associated with a substantial risk of delayed major bleeding that varies with the type of procedure [22]. HBT increases the risk of bleeding without providing clinical benefit to these patients. However, all included many types of endoscopic procedures without focusing on specific procedures or organs [21, 22].

Hence, in the present study, we conducted a nationwide multicenter study in Japan, and examined a large number of patients receiving DOACs and warfarin, to compare the clinical efficacy of the various DOACs and conventional warfarin treatment on post-gastric ESD bleeding.

\section{Materials and methods}

\section{Patients}

We conducted a multicenter, retrospective study at 25 institutions in Japan. Data were included of 728 consecutive patients receiving anticoagulants, i.e., warfarin or DOACs, who underwent ESD for gastric neoplasms between November 2011 and October 2016. All indicated lesions were preoperatively confirmed to be adenocarcinomas or suspected adenocarcinomas. We excluded the following cases: (1) administration of anticoagulants was not resumed after ESD; (2) the ESD procedure was not completed; (3) ESD for other upper gastrointestinal neoplasia was performed within 30 days after the gastric ESD; (4) additional surgical resection was performed within 30 days after the ESD and (5) cases had missing data.

All authors had access to the study data and reviewed and approved the final manuscript. This study was carried out in accordance with the Declaration of Helsinki and was approved by the institutional review board of the participating institutions (The Ethics Committee of Ehime Prefectural Central Hospital Registry number: 28-79). Written informed consent was obtained from all patients enrolled in this study.

\section{ESD procedure}

Performance of ESD was principally indicated for possible node-negative EGC, following criteria described by Gotoda et al. [1]. ESD was performed according to standard procedures. Briefly, the procedure included: (1) marking around the lesion; (2) submucosal injection of $0.2-0.4 \%$ sodium hyaluronic acid solution; (3) application of a mucosal incision outside of the marked region and submucosal dissection with a cutting device; (4) hemostasis of active bleeding, as well as prophylactic coagulation of visible vessels on the mucosal defect using hemostatic forceps or placement of clips either during submucosal dissection or during the final step of ESD. In some cases, the mucosal defect was closed using an endoloop and endoclips when completing the ESD procedure [23]. In other cases, the mucosal defect was covered with polyglycolic acid (PGA) sheets and fibrin glue during the completion of the ESD procedure [24].

\section{Management of anticoagulants and antiplatelet agents}

The administered anticoagulants included warfarin and DOACs such as dabigatran, rivaroxaban, apixaban and edoxaban. Antiplatelet agents provided to the patients included aspirin, cilostazol, ticlopidine and clopidogrel. 
The duration of withdrawal of antithrombotic agents were determined according to the guidelines published in 2014 by the Japan Gastroenterological Endoscopy Society (JGES) [12]: 3-5 days for warfarin, 1-2 days for DOACs, three to 5 days for aspirin, 1 day for cilostazol, and 5-7 days for ticlopidine and clopidogrel before the procedure, and were resumed 1 day after ESD. Patients who received anticoagulant therapy preoperatively were recommended for HBT as per the JGES guidelines from 2014. However, patients who were considered to have a low risk of thromboembolism, for instance, based on the CHADS2 or CHA2DS2-VASc score, were excluded from HBT [25]. The decision to discontinue anticoagulants or to perform HBT in patients undergoing ESD was based on the judgment of the prescribing physician and endoscopists.

\section{Adverse events}

Delayed bleeding was defined as an event requiring emergency endoscopy with endoscopic hemostasis or transfusion for the management of hematemesis or melena, or a decrease $>2 \mathrm{~g} / \mathrm{dL}$ of hemoglobin levels after ESD. This definition did not include cases of preventive hemostasis for exposed vessels on the mucosal defect without the clinical criterion of bleeding on second-look endoscopy.

\section{Statistical analysis}

All continuous variables were expressed as median and range. Statistical analyses were performed using Fisher exact tests, Mann-Whitney $U$ tests, log-rank tests or the Kaplan-Meier method, as appropriate. We used Cochran-Armitage tests to assess correlations between the number of antiplatelets combined with anticoagulants and delayed bleeding rate. Multivariate logistic regression models were used to examine the effect of independent variables on delayed bleeding. Multivariate models included factors suggested in studies associated with delayed bleeding and those that were statistically significant $(p<0.05)$ in univariate analysis. To reduce confounding effects of covariates, we used logistic regression to perform propensity score matching (PSM) with the following independent variables: gender, age, chronic kidney disease, lesion size, cessation of anticoagulant, and concomitant use of antiplatelet agent. Patients receiving dabigatran were matched with those receiving other DOACs according to the generated PSM using a caliper width of 0.2 . On the completion of matching, we examined the influence of DOACs on delayed bleeding. A $p$ value $<0.05$ was considered as statistically significant. Statistical analyses were performed using the EZR version 1.41 , which is a graphical user interface for $\mathrm{R}$ (The R Foundation for Statistical Computing, Vienna, Austria).

\section{Results}

\section{Characteristics of patients and lesions}

We treated 849 gastric neoplasms by gastric ESD in 728 patients, who were being treated with anticoagulant medication, 467 of whom received warfarin and 261 of whom received DOACs. Of the latter group, 92 patients received dabigatran, 103 rivaroxaban, 45 apixaban and 21 edoxaban. Twenty-three percent of the patients received antiplatelet agents.

Characteristics of the patients and their lesions are shown in Table 1 . The median age of the patients was 76 years (range $48-90$ years), and $86 \%$ were males. The proportion of males among DOAC-treated patients was higher than that in the warfarin-treated group. The frequency of atrial fibrillation (AF) was higher in the DOAC group than in the warfarin group, because DOACs are prescribed for non-valvular AF and deep-vein thrombosis based on standards implemented in the Japanese health insurance system. In addition, patients in the DOAC group had fewer severe comorbidities (e.g., ischemic heart disease) than those in the warfarin group. Therefore, antiplatelet agents were used less often in the DOAC group. However, the median CHADS2 and CHA2DS2-VASc scores were identical in the two groups and no betweengroup difference was found in lesions.

\section{Perioperative managements of gastric ESD}

The proportions of patients receiving HBT in the warfarin- and DOAC groups were $60 \%$ and $16 \%$, respectively (Table 2). Although JGES guidelines recommend the application of HBT to patients treated with warfarin, ESD was carried out without HBT in $36 \%$ of these patients, and with uninterrupted warfarin therapy maintained in $4 \%$ of the patients. Thirty percent (37/122) of patients on warfarin therapy and $26 \%(11 / 31)$ of patients on DOACs continued their intake of antiplatelet agents on the day of ESD, although $21 \%$ (9/42) of the patients on thienopyridine derivatives were given replacement with aspirin or cilostazol during the perioperative period.

The proportions of patients who received endoscopic closure or tissue-shielding method with PGA sheets and fibrin glue to prevent delayed bleeding were 3 and $7 \%$, respectively, in the warfarin group, and 3 and $6 \%$ in the DOAC-treated group. There were no differences between the two groups in the proportion of patients treated with proton-pump inhibitors (PPI) or mucosa-protecting agents after ESD, or with intravenous PPI on the day of the ESD procedure. 
Table 1 Characteristics of patients and lesions according to the type of anticoagulant

\begin{tabular}{|c|c|c|c|}
\hline & Warfarin $(n=467)$ & DOACs $(n=261)$ & $p$ value \\
\hline \multicolumn{4}{|l|}{ Patients } \\
\hline Age, median (range), years & $76(64-88)$ & $74(53-84)$ & 0.11 \\
\hline Gender (male/female), $n$ & $394 / 73$ & $234 / 27$ & 0.04 \\
\hline Antiplatelets $(0 / 1 / 2), n$ & $345 / 104 / 18$ & $219 / 42 / 0$ & $<0.01$ \\
\hline \multicolumn{4}{|l|}{ Comorbidities, $n(\%)$} \\
\hline Atrial Fibrillation & $328(70)$ & $232(89)$ & $<0.01$ \\
\hline Deep vein thrombosis & $25(5.4)$ & $7(2.7)$ & 0.09 \\
\hline Cardiac valve replacement & $22(4.7)$ & $9(3.4)$ & 0.41 \\
\hline Congestive heart failure & $119(25)$ & $55(21)$ & 0.18 \\
\hline Hypertension & $297(64)$ & $142(54)$ & 0.01 \\
\hline Diabetes mellitus & $133(28)$ & $62(24)$ & 0.16 \\
\hline Prior stroke or transient ischemic attacks & $101(22)$ & $44(17)$ & 0.12 \\
\hline Ischemic heart disease & $117(25)$ & $41(16)$ & $<0.01$ \\
\hline Chronic kidney disease & $168(36)$ & $88(34)$ & 0.54 \\
\hline CHADS2 score, median (range) ${ }^{\mathrm{a}}$ & $2(0-6)$ & $2(0-6)$ & \\
\hline Thrombotic risk (low; score $\leq 2 /$ moderate or high; score $\geq 3$ ) & $293 / 174$ & $179 / 82$ & 0.12 \\
\hline CHA2DS2-VASc score, median (range) ${ }^{\mathrm{a}}$ & $3(0-8)$ & $3(0-7)$ & \\
\hline Thrombotic risk (low; score $\leq 4$ /moderate or high; score $\geq 5$ ) & $352 / 115$ & $213 / 48$ & 0.06 \\
\hline \multicolumn{4}{|l|}{ Lesions } \\
\hline Number of resected lesion $(1 / 2 / 3<), n$ & $404 / 48 / 15$ & $231 / 23 / 7$ & 0.44 \\
\hline Lesion size, median (range), mm & $15(1-122)$ & $15(1-60)$ & 0.42 \\
\hline Lesion location (U/M/L), $n$ & $85 / 198 / 184$ & $36 / 120 / 105$ & 0.29 \\
\hline Macroscopic type (Protruding/Flat/Depressed), $n$ & $255 / 10 / 198$ & $110 / 7 / 142$ & $<0.01$ \\
\hline Ulceration in lesion $( \pm), n$ & $30 / 432$ & $15 / 245$ & 0.69 \\
\hline Histology (differentiated/undifferentiated/adenoma), $n$ & $408 / 11 / 44$ & $242 / 6 / 12$ & 0.06 \\
\hline Depth of invasion in cancer (mucosa/submucosa), $n$ & $361 / 58$ & $215 / 33$ & 0.90 \\
\hline
\end{tabular}

${ }^{\text {a }}$ Patients with atrial fibrillation

DOACs direct oral anticoagulants, $U$ upper third of stomach, $M$ middle third of stomach, $L$ lower third of stomach

All $p$ values less than 0.05 were shown in bold

\section{Treatment outcome of the DOACs and warfarin groups}

The rate of delayed bleeding in the DOAC group was not different from that in the warfarin group (Table 2). In comparisons between individual agents, delayed bleeding rates were different for each agent (Fig. 1a). In patients taking dabigatran, the delayed bleeding rate was the lowest of patients taking anticoagulants. Furthermore, the delayed bleeding rate in dabigatran-treated patients was lower than that of warfarin-treated ones ( $8 \%$ vs $18 \%, p=0.018$ ), and lower than that of patients treated with other DOACs ( $8 \%$ vs $17 \%$, $p=0.033$; Fig. 1b). In warfarin-treated patients, delayed bleeding was more frequently seen among those receiving HBT. Compared with patients who did not receive HBT, the delayed-bleeding rate was higher in patients undergoing HBT, and higher yet in patients receiving continued anticoagulant therapy without cessation, and this occurred equally in the warfarin and in the DOAC Group (Fig. 1c). The amount of time between the ESD and the onset of delayed bleeding did not differ between warfarin- and DOAC groups (Fig. 1b), and was the same regardless of whether patients underwent HBT or not. In regard to the combined use of antiplatelet agents, delayed bleeding rates of patients receiving either no antiplatelet agent, a single antiplatelet agent, or dual antiplatelet agents were $12 \%, 27 \%$, and $50 \%$, respectively. It was observed that the delayed bleeding rate rose as the number of antiplatelet agents increased $(p<0.001$; Fig. 1d). In patients who had undergone closure of mucosal defect using endoloop and endoclips, delayed bleeding occurred in two out of the 22 cases (9\%; Fig. 1e), which did not differ significantly from patients who did not receive these preventive measures. However, notably, 18 patients with complete closures of the mucosal defect did not develop delayed bleeding, although two out of four patients with incomplete closures showed delayed bleeding. However, in patients who underwent preventative procedures using PGA sheets and fibrin glue to cover their mucosal defect, delayed 
Table 2 Perioperative management and treatment outcome of gastric ESD for different anticoagulants

\begin{tabular}{|c|c|c|c|}
\hline & Warfarin $(n=467)$ & DOACs $(n=261)$ & $p$ value \\
\hline Management of anticoagulants & & & $<0.01$ \\
\hline Cessation (without HBT), $n(\%)$ & $166(36)$ & $206(79)$ & \\
\hline Heparin bridging therapy, $n(\%)$ & $280(60)$ & $41(16)$ & \\
\hline Continuation (Without cessation), $n(\%)$ & $21(4)$ & $4(5)$ & \\
\hline Management of antiplatelets & & & 0.69 \\
\hline Cessation, $n(\%)$ & $85(18)$ & $31(12)$ & \\
\hline Continuation (without cessation), $n(\%)$ & $37(8)$ & $11(4)$ & \\
\hline \multicolumn{4}{|l|}{ Perioperative managements } \\
\hline Closure of mucosal defect, $n(\%)$ & $15(3)$ & $7(3)$ & 0.68 \\
\hline Covering mucosal defect with PGA sheet, $n(\%)$ & $32(7)$ & $15(6)$ & 0.56 \\
\hline Gastric acid inhibitor, $n(\%)$ & & & 0.56 \\
\hline Proton pump inhibitor & $421(90)$ & $226(87)$ & \\
\hline Potassium competitive acid blocker & $40(9)$ & $35(13)$ & \\
\hline Intravenous PPI, $n(\%)$ & $366(78)$ & $193(74)$ & 0.17 \\
\hline Mucosa protecting agents, $n(\%)$ & $272(58)$ & $146(56)$ & 0.54 \\
\hline \multicolumn{4}{|l|}{ Treatment outcome } \\
\hline En bloc resection, $n(\%)$ & $465(99)$ & $260(99)$ & 1 \\
\hline Delayed bleeding, $n(\%)$ & $82(18)$ & $36(14)$ & 0.20 \\
\hline With/without HBT, $n$ & $55 / 27$ & $8 / 28$ & $<0.01$ \\
\hline Periods until delayed bleeding, median (range), day & $6(0-18)$ & $7(0-29)$ & 0.75 \\
\hline With/without HBT, median, day & $6 / 8$ & $7 / 10$ & 0.12 \\
\hline Blood transfusion, $n(\%)$ & $35(7)$ & $5(2)$ & $<0.01$ \\
\hline Perforation, $n(\%)$ & $6(1)$ & $2(1)$ & 0.71 \\
\hline Thrombotic event, $n(\%)$ & 0 & 0 & 1 \\
\hline Period of HBT after ESD, median (range) day & $5(1-23)$ & $2(1-12)$ & $<0.01$ \\
\hline Hospitalization period, median (range), day & $13(4-53)$ & $9(4-32)$ & $<0.01$ \\
\hline
\end{tabular}

$H B T$ heparin bridging therapy, $P G A$ polyglycolic acid, $P P I$ proton pump inhibitor, $E S D$ endoscopic submucosal dissection

All $p$ values less than 0.05 were shown in bold bleeding occurred in 10 of 47 cases (21\%), which was not different from the patients who did not receive these preventive treatments.

\section{Clinical risk factors for delayed bleeding}

We compared the lesions and characteristics of patients to those without delayed bleeding. Univariate analysis showed that an increased risk of delayed bleeding was associated with having an age $>65$ years, being male, receiving $\mathrm{HBT}$ after ESD, being treated with combined antiplatelet agents, resection of multiple lesions and the presence of lesions $>20 \mathrm{~mm}$ (Table 3). Both Dabigatran treatment and cessation of anticoagulants without HBT reduced the risk of delayed bleeding. In multivariate analysis, age $>65$ years (OR 2.96; 95\% CI 1.13-7.73), being treated with combined antiplatelet agents (OR 2.70; 95\% CI 1.74-4.21), being male (OR 2.12; 95\% C 1.01-4.45) and lesions $>20 \mathrm{~mm}$ (OR 1.67; 95\% CI 1.08-2.59) were independent risk factors for delayed bleeding, whereas cessation of anticoagulants without HBT was associated with a low risk of delayed bleeding (OR $0.32 ; 95 \% \mathrm{CI}$ 0.14-0.76).

Patients with bleeding within 6 days after ESD and those with bleeding after 7 days of ESD were compared with patients without bleeding (Supplementary Tables 1 and 2 ). In the early bleeding group, presence of ulceration (OR 2.74 ; 95\% CI 1.08-6.97), being treated with combined antiplatelet agents (OR 2.15; 95\% CI 1.19-3.89), lesions in the lower third of the stomach (OR 2.07; 95\% CI 1.18-3.63), and lesions of $>20 \mathrm{~mm}$ (OR 1.85; 95\% CI 1.05-3.27) were independent risk factors for delayed bleeding, and cessation of anticoagulants without HBT was associated with a low risk of delayed bleeding (OR 0.42; 95\% CI 0.23-0.76). In the late bleeding group, being treated with combined antiplatelet agents was the only independent risk factor for delayed bleeding (OR 3.17; 95\% CI 1.80-5.58).

We also conducted the univariate and multivariate analysis for delayed bleeding exclusively in patients receiving DOACs, to assess the optimal, appropriate use of DOACs. 

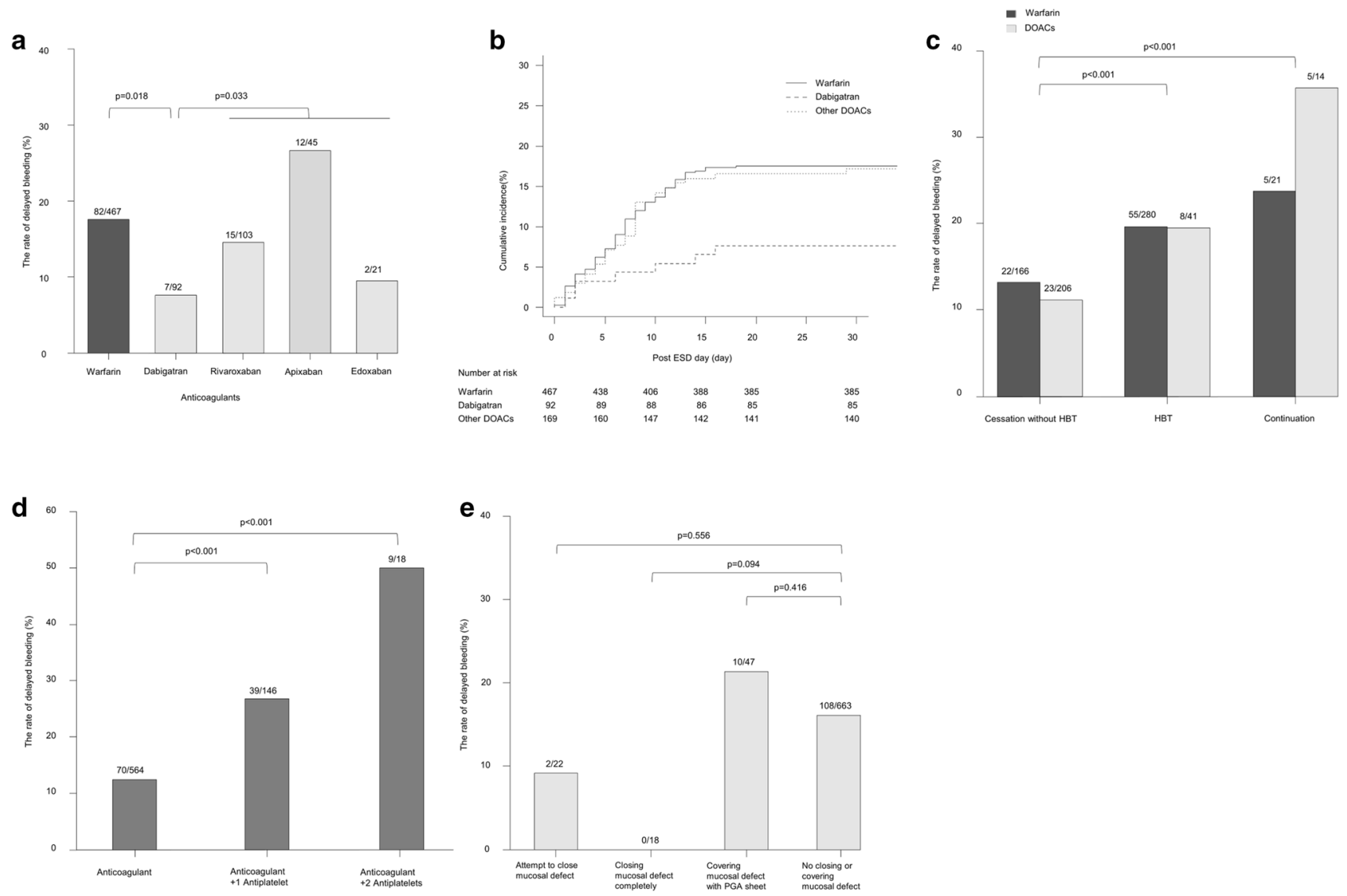

Fig. 1 a The delayed-bleeding rates in patients receiving warfarin and DOACs. b Time-to-event curve of delayed bleeding after gastric ESD in the different patient groups. $\mathbf{c}$ The delayed-bleeding rates in patients without HBT, patients with HBT and patients receiving continuous anticoagulants. $\mathbf{d}$ Rates of delayed bleeding in patients receiv- ing only anticoagulants, one antiplatelet combined with an anticoagulant, and two antiplatelets combined with an anticoagulant. e Rates of delayed bleeding in patients who attempted to close mucosal defect, in whom defect was closed completely, in whom defect covered with PGA sheet, and in those who did not undergo both the methods
Dabigatran and cessation of DOACs were independent lowrisk factors for delayed bleeding after ESD (Table 4).

To identify differences of delayed-bleeding rates between dabigatran and other DOACs, we applied a PSM procedure (Supplementary Table 3). Ninety-one matched dabigatranother DOAC patient pairs were formed. The two groups did not differ in terms of patient characteristics or lesions. However, the delayed bleeding rate of the patients taking dabigatran was significantly lower than that of patients receiving other DOACs.

\section{Discussion}

In the present study, we examined the efficacies of DOACs in gastric ESD patients by comparisons with warfarin treatment in a large cohort of 728 patients from institutions across Japan. This is the largest study of gastric ESD to date in patients treated with anticoagulant therapy, partly because the ratio of patients taking anticoagulants among all gastric
ESD cases is only $2.3 \%-5.7 \%[5,7,8]$. However, the number of patients taking anticoagulants is surely expected to rise with the ongoing aging of the society, and the proportion of DOAC users in this study has been also steadily increased from year to year (Supplementary Fig. 1). Therefore, this study may contribute to the perioperative management of anticoagulants to reduce delayed bleeding after ESD of EGC in patients treated with anticoagulants.

We previously reported that the delayed bleeding risk of patients receiving dabigatran was significantly lower than those treated with rivaroxaban because of different pharmacokinetics of the two drugs. However, in the previous study, the number of patients receiving DOACs was small [9]. In this present study, dabigatran was again shown to significantly lower delayed-bleeding risk relative to other DOACs, as confirmed by both univariate and multivariate analyses (Table 4). In a PSM analysis conducted to reduce selection bias in patients, the rate of delayed bleeding in patients on dabigatran was significantly lower than that in patients receiving other DOACs (Supplementary Table 3). 
Table 3 Univariate and multivariate analysis of risk factors for delayed bleeding after gastric ESD

\begin{tabular}{|c|c|c|c|c|c|c|}
\hline & \multicolumn{3}{|c|}{ Univariate analysis } & \multicolumn{3}{|c|}{ Multivariate analysis } \\
\hline & Odds ratio & $95 \% \mathrm{CI}$ & $p$ value & Odds ratio & $95 \% \mathrm{CI}$ & $p$ value \\
\hline Age $(\geq 65$ years $)$ & 2.97 & $1.17-9.65$ & 0.01 & 2.96 & $1.13-7.73$ & 0.02 \\
\hline Gender (male) & 2.12 & $1.02-4.94$ & $\mathbf{0 . 0 3}$ & 2.12 & $1.01-4.45$ & 0.04 \\
\hline \multicolumn{7}{|l|}{ Anticoagulants } \\
\hline Warfarin & 1.33 & $0.86-2.10$ & 0.21 & & & \\
\hline Dabigatran & 0.39 & $0.15-0.87$ & 0.01 & 0.49 & $0.21-1.15$ & 0.10 \\
\hline Rivaroxaban & 0.86 & $0.45-1.58$ & 0.77 & & & \\
\hline Apixaban & 1.97 & $0.90-4.08$ & 0.05 & & & \\
\hline Edoxaban & 0.53 & $0.06-2.27$ & 0.55 & & & \\
\hline Cessation of anticoagulants without HBT & 0.53 & $0.35-0.81$ & $<0.01$ & 0.32 & $0.14-0.76$ & 0.01 \\
\hline Heparin bridging therapy after ESD & 1.56 & $1.03-2.37$ & $\mathbf{0 . 0 3}$ & & & \\
\hline Antiplatelets (concomitant use) & 2.91 & $1.87-4.52$ & $<0.01$ & 2.70 & $1.74-4.21$ & $<0.01$ \\
\hline Continued use of antiplatelets & 0.86 & $0.37-1.91$ & 0.85 & 0.51 & $0.22-1.17$ & 0.11 \\
\hline Resection of multiple lesions $(\geq 2)$ & 2.00 & $1.14-3.41$ & 0.01 & 1.73 & $0.99-3.01$ & 0.05 \\
\hline Lesion size $(>20 \mathrm{~mm})$ & 1.82 & $1.18-2.80$ & $<0.01$ & 1.67 & $1.08-2.59$ & 0.02 \\
\hline Location (lower third of stomach) & 1.46 & $0.96-2.21$ & 0.06 & 1.51 & $0.98-2.30$ & 0.05 \\
\hline Macroscopic type (depressed type) & 1.06 & $0.69-1.64$ & 0.83 & & & \\
\hline Presence of ulceration & 1.72 & $0.76-3.61$ & 0.14 & 2.02 & $0.91-4.43$ & 0.08 \\
\hline Histology (differentiated type) & 1.49 & $0.34-13.7$ & 1 & & & \\
\hline Depth of invasion (mucosa) & 1.10 & $0.59-2.19$ & 0.87 & & & \\
\hline Gastric acid inhibitor (P-cab) & 1.21 & $0.60-2.29$ & 0.51 & & & \\
\hline Intravenous PPI & 1.15 & $0.70-1.94$ & 0.63 & & & \\
\hline Mucosa protecting agents & 1.30 & $0.85-2.00$ & 0.22 & & & \\
\hline Closing mucosal defect & 0.50 & $0.05-2.14$ & 0.56 & & & \\
\hline Covering mucosal defect with PGA sheet & 1.43 & $0.62-3.05$ & 0.31 & & & \\
\hline
\end{tabular}

$H B T$ heparin bridging therapy, $E S D$ endoscopic submucosal dissection, $P-c a b$ potassium competitive acid blocker, $P P I$ proton pump inhibitor, $P G A$ polyglycolic acid

All $p$ values less than 0.05 were shown in bold

DOACs have different properties from warfarin, but individual DOACs have distinct pharmacological properties. Warfarin targets vitamin $\mathrm{K}$ epoxide reductase in the liver to block the function of the coagulation factors II, III, IX, and X. In contrast to warfarin, which exerts a systemic anticoagulant effect, DOACs directly target coagulation proteins and have both systemic and local effects on the gastric mucosa, with the exception of dabigatran. Dabigatran is administrated as a prodrug that does not exhibit anticoagulant activity and is converted to its active form by hepatic and serum esterases [26]. Therefore, dabigatran may not have local anticoagulant effect in the stomach, whereas other DOACs may have direct anticoagulant effect in postgastric ESD ulcer sites and potentially induce local bleeding $[25,27]$. These differences may explain the variance in the risk of delayed bleeding between warfarin and DOACs, and between dabigatran and other DOACs.

It was also reported previously that dabigatran is more effective than other DOACs in inducing clotting after vascular injury [28]. This observation was consistent with other studies that showed a low rate of intracranial hemorrhage during dabigatran therapy [29] and a low rate of bleeding complications during catheter ablation in patients receiving dabigatran [30]. We suspect that a similar response to vascular injury of the post-ESD ulcers could support favorable outcomes, reflected by a difference in bleeding risk between patients treated with dabigatran or other DOACs.

Although the number of patients who received continued anticoagulants during the perioperative period was limited, continuation of warfarin and DOACs was associated with higher delayed-bleeding rates, i.e., 24\% (5/21) in warfarintreated- and 36\% (5/14) in DOAC-treated patients (Fig. 1c). Thus, for the patients on DOACs, a one- or two-day therapeutic discontinuation without HBT would be appropriate because no higher risk of delayed bleeding or thrombosis would occur. For patients on warfarin, a continuation of warfarin treatment would seem a good option, because warfarin requires 5 days of discontinuation before diminishing its reported effects to cause one percent of thrombotic event [31] or a nine-percent (2/22) higher rate of delayed bleeding, 
Table 4 Multivariate analysis of risk factors for delayed bleeding after gastric ESD in patients taking DOACs

\begin{tabular}{|c|c|c|c|c|c|c|}
\hline & \multicolumn{3}{|c|}{ Univariate analysis } & \multicolumn{3}{|c|}{ Multivariate analysis } \\
\hline & Odds ratio & $95 \% \mathrm{CI}$ & $p$ value & Odds ratio & $95 \% \mathrm{CI}$ & $p$ value \\
\hline Age ( $\geq 65$ years $)$ & 3.59 & $0.54-153$ & 0.32 & & & \\
\hline Gender (male) & 2.12 & $0.48-19.2$ & 0.39 & & & \\
\hline \multicolumn{7}{|l|}{ DOACs } \\
\hline Dabigatran & 0.39 & $0.14-0.98$ & $\mathbf{0 . 0 3}$ & 0.40 & $0.16-0.97$ & 0.04 \\
\hline Other DOACs & 2.50 & $1.01-7.08$ & $\mathbf{0 . 0 3}$ & & & \\
\hline High dosage of DOACs & 0.72 & $0.33-1.56$ & 0.36 & & & \\
\hline Cessation of anticoagulants without HBT & 0.40 & $0.18-0.95$ & 0.02 & 0.40 & $0.18-0.87$ & 0.02 \\
\hline Heparin bridging therapy after ESD & 1.65 & $0.60-4.15$ & 0.32 & & & \\
\hline Antiplatelets (concomitant use) & 1.93 & $0.73-4.72$ & 0.14 & 1.47 & $0.60-3.55$ & 0.39 \\
\hline Continued use of antiplatelets & 2.45 & $0.39-10.9$ & 0.18 & & & \\
\hline Resection of multiple lesions $(\geq 2)$ & 1.28 & $0.36-3.79$ & 0.58 & & & \\
\hline Lesion size (>20 mm) & 1.80 & $0.79-3.97$ & 0.11 & 1.85 & $0.86-3.96$ & 0.11 \\
\hline Location (lower third of stomach) & 1.22 & $0.55-2.63$ & 0.58 & & & \\
\hline Macroscopic type (depressed type) & 1.05 & $0.49-2.30$ & 1 & & & \\
\hline Presence of ulceration & 0.95 & $0.10-4.51$ & 1 & & & \\
\hline Depth of invasion (mucosa) & 1.18 & $0.37-4.93$ & 1 & & & \\
\hline Gastric acid inhibitor (P-cab) & 1.35 & $0.42-3.69$ & 0.59 & & & \\
\hline Intravenous PPI & 1.53 & $0.61-4.38$ & 0.41 & & & \\
\hline Mucosa protecting agents & 1.11 & $0.51-2.46$ & 0.85 & & & \\
\hline
\end{tabular}

$D O A C s$ direct oral anticoagulants, ESD endoscopic submucosal dissection, $H B T$ heparin bridging therapy, $P$-cab potassium competitive acid blocker, $P P I$ proton pump inhibitor

All $p$ values less than 0.05 were shown in bold

as reported recently [32]. A larger study is needed to assess the actual risk of continuing warfarin when carrying out ESD procedures. When the patients receiving anticoagulant treatment have a low risk of thrombosis, e.g., a history of deep-vein thrombus or AF with a CHADS2 score of 1 and they could discontinue it, these patients significantly had a low risk of delayed bleeding in multivariate analysis (Tables 3,4), although the patients rarely can discontinue anticoagulant treatment during the perioperative period [33]. HBT was still performed, but especially in warfarin-treated patients delayed bleeding occurred significantly more often in patients who received HBT than in those who were not (Fig. 1c), an observation that confirmed results of previous studies [7, 9, 14, 33]. Although former Japanese guidelines recommended HBT for warfarin-treated patients, new guidelines issued in 2017 do no longer recommend HBT and merely suggest whether to continue warfarin treatment or switching to DOACs without HBT [34] backed by sufficient supportive data.

Three guidelines that are currently available suggest the of implementation of a drug withdrawal period from a pharmacological perspective and do not reflect the results of clinical studies on ESD [35-37]. Although thromboembolism is serious and potentially fatal, gastrointestinal bleeding is often controllable, and measures to control bleeding while maintaining anticoagulant effectiveness should be considered.

With respect of how to deal with ongoing anticoagulant treatments to minimize the risk of delayed bleeding, a reasonable option would be to switch current anticoagulant treatment to dabigatran, especially after discontinuation of apixaban, rivaroxaban, and warfarin, for a one-month long postoperative period after ESD, because the delayed bleeding rate of dabigatran (8\%) is lower than that of apixaban (27\%), rivaroxaban (15\%), and even warfarin (18\%; Fig. 1a). We consider dabigatran to be the better alternative for warfarin and other DOACs because of the results of the present study.

In some patients, tissue shielding using a PGA sheet and fibrin glue [24], and closure of mucosal defects using endoloop and endoclips [23] were performed to prevent delayed bleeding. However, bleeding was not significantly reduced in comparison with patients who did not undergo these procedures (Fig. 1e). The PGA-shielding method showed no effect on the prevention of delayed bleeding, confirming another recent report [38]. The complete closure of mucosal defects was achieved in $82 \%(18 / 22)$ of patients in whom such closures were attempted and the patients who did not show mucosal defects had no delayed bleeding. The closure of mucosal defects itself could be 
useful; however, the success to close all mucosal defects critically depends on the location and the size. Although we still have to overcome a number of technical difficulties, closing the mucosal defect could be a good countermeasure to prevent post-bleeding in patients receiving antithrombotic agents.

A number of limitations of the current study should be noted. The first is that this was a retrospective study, albeit of a large number of cases in treated in multiple centers. Second, the management of anticoagulants varied in several ways, especially for DOACs, because there was no statement about DOACs other than the use of dabigatran in the guidelines [12] during the examined period. Thirdly, we could not conduct a systematic evaluation of the effect of edoxaban, because the number of included patients receiving edoxaban was insufficient. Fourth, the risk of thromboembolism could not be examined, because no case with a thrombotic event emerged.

In conclusion, this is the first study to directly compare and clarify the effects of DOACs surrounding endoscopic treatment in a large number of patients. Patients taking oral anticoagulants had a higher risk of delayed bleeding after gastric ESD, but the delayed bleeding rate varied with the specific type of drug administered. In the present study, dabigatran was associated with a lower rate of delayed bleeding than other anticoagulants, i.e., warfarin and other DOACs. Although this was a retrospective study, its multicenter character and the large number of cases included in the analysis support the validity of the present results. The superior efficacy of dabigatran to reduce delayed bleeding after gastric ESD should be demonstrated in future prospective studies. Changing oral anticoagulants to dabigatran in the perioperative period could be a good treatment choice for reducing delayed bleeding after gastric ESD.

Acknowledgements We thank Naomi Kakushima (Shizuoka Cancer Center) and Itaru Saitou (The University of Tokyo) for enrollment of the patients and for the data collection; and we thank Mary Raina Angeli Abad (Showa University Koto Toyosu Hospital) for Englishlanguage editing.

Author contributions Planning and conducting the study: HT and TY; collecting data: HT, TY, KI, YM, IO, TI, TH, TS, HD, YT, JN, WH, TM, MI, KS, KY, KK, SK, WH, AY, TU, TY, KH, KK, TF, and TN; interpreting data: HT, TY, NK, YM, TH, HD, WH, KY, TN and MF; drafting the manuscript: HT; critical revision of the manuscript: TY and MF; statistical analysis: HT; study supervision: TY and MF. All authors listed have contributed substantially to the design, data collection and analysis, as well as to the editing of the manuscript.

Funding None.

\section{Compliance with ethical standards}

Conflict of interest The authors declare that they have no conflict of interest.

\section{References}

1. Gotoda T, Yanagisawa A, Sasako M, Ono H, Nakanishi Y, Shimoda $\mathrm{T}$, et al. Incidence of lymph node metastasis from early gastric cancer: estimation with a large number of cases at two large centers. Gastric Cancer. 2000;3:219-25.

2. Kato M, Nishida T, Yamamoto K, Hayashi S, Kitamura S, Yabuta T, et al. Scheduled endoscopic surveillance controls secondary cancer after curative endoscopic resection for early gastric cancer: a multicentre retrospective cohort study by Osaka University ESD study group. Gut. 2013;62:1425-32.

3. Takizawa K, Oda I, Gotoda T, Yokoi C, Matsuda T, Saito Y, et al. Routine coagulation of visible vessels may prevent delayed bleeding after endoscopic submucosal dissection-an analysis of risk factors. Endoscopy. 2008;40:179-83.

4. Okada K, Yamamoto Y, Kasuga A, Omae M, Kubota M, Hirasawa T, et al. Risk factors for delayed bleeding after endoscopic submucosal dissection for gastric neoplasm. Surg Endosc. 2011;25:98-107.

5. Koh R, Hirasawa K, Yahara S, Oka H, Sugimori K, Morimoto $\mathrm{M}$, et al. Antithrombotic drugs are risk factors for delayed postoperative bleeding after endoscopic submucosal dissection for gastric neoplasms. Gastrointest Endosc. 2013;78:476-83.

6. Takeuchi T, Ota K, Harada S, Edogawa S, Kojima Y, Tokioka $\mathrm{S}$, et al. The postoperative bleeding rate and its risk factors in patients on antithrombotic therapy who undergo gastric endoscopic submucosal dissection. BMC Gastroenterol. 2013;13:136.

7. Yoshio T, Nishida T, Kawai N, Yuguchi K, Yamada T, Yabuta $\mathrm{T}$, et al. Gastric ESD under heparin replacement at high-risk patients of thromboembolism is technically feasible but has a high risk of delayed bleeding: Osaka University ESD Study Group. Gastroenterol Res Pract. 2013;2013:365830.

8. Furuhata T, Kaise M, Hoteya S, Iizuka T, Yamada A, Nomura $\mathrm{K}$, et al. Postoperative bleeding after gastric endoscopic submucosal dissection in patients receiving antithrombotic therapy. Gastric Cancer. 2017;20:207-14.

9. Yoshio T, Tomida H, Iwasaki R, Horiuchi Y, Omae M, Ishiyama A. Effect of direct oral anticoagulants on the risk of delayed bleeding after gastric endoscopic submucosal dissection. Dig Endosc. 2017;29:686-94.

10. Yoshio T, Nishida T, Hayashi Y, Iijima H, Tsujii M, Fujisaki J, et al. Clinical problems with antithrombotic therapy for endoscopic submucosal dissection for gastric neoplasms. World J Gastrointest Endosc. 2016;8:756-62.

11. Numata N, Oka S, Tanaka S, Higashiyama M, Sanomura Y, Yoshida S, et al. Clinical outcomes of endoscopic submucosal dissection for early gastric cancer in patients with chronic kidney disease. J Gastroenterol Hepatol. 2013;28:1632-7.

12. Fujimoto K, Fujishiro M, Kato M, Higuchi K, Iwakiri R, Sakamoto C, et al. Japan Gastroenterological Endoscopy Society. Guidelines for gastroenterological endoscopy in patients undergoing antithrombotic treatment. Dig Endosc. 2014;26:1-14.

13. Beyer-Westendorf J, Gelbricht V, Förster K, Ebertz F, Köhler $\mathrm{C}$, Werth S, et al. Peri-interventional management of novel oral anticoagulants in daily care: results from the prospective Dresden NOAC registry. Eur Heart J. 2014;35:1888-96.

14. Shindo Y, Matsumoto S, Miyatani H, Yoshida Y, Mashima H. Risk factors for postoperative bleeding after gastric endoscopic submucosal dissection in patients under antithrombotics. World J Gastrointest Endosc. 2016;8:349-56.

15. Siegal D, Yudin J, Kaatz S, Douketis JD, Lim W, Spyropoulos AC. Periprocedural heparin bridging in patients receiving vitamin $\mathrm{K}$ antagonists: systematic review and meta-analysis of bleeding and thromboembolic rates. Circulation. 2012;126:1630-9. 
16. Douketis JD, Healey JS, Brueckmann M, Eikelboom JW, Ezekowitz MD, Fraessdorf M, et al. Perioperative bridging anticoagulation during dabigatran or warfarin interruption among patients who had an elective surgery or procedure. Substudy of the RE-LY trial. Thromb Haemost. 2015;113:625-32.

17. Douketis JD, Spyropoulos AC, Kaatz S, Becker RC, Caprini JA, Dunn AS, et al. Perioperative bridging anticoagulation in patients with atrial fibrillation. N Engl J Med. 2015;373:823-33.

18. Caldeira D, Barra M, Ferreira A, Rocha A, Augusto A, Pinto FJ, et al. Systematic review with meta-analysis: the risk of major gastrointestinal bleeding with non-vitamin K antagonist oral anticoagulants. Aliment Pharmacol Ther. 2015;42:1239-49.

19. Yao X, Abraham NS, Sangaralingham LR, Bellolio MF, McBane $\mathrm{RD}$, Shah ND, et al. Effectiveness and safety of dabigatran, rivaroxaban, and apixaban versus warfarin in nonvalvular atrial fibrillation. J Am Heart Assoc. 2016;5:e003725.

20. Lip GYH, Keshishian A, Li X, Hamilton M, Masseria C, Gupta $\mathrm{K}$, et al. Effectiveness and safety of oral anticoagulants among nonvalvular atrial fibrillation patients. Stroke. 2018;49:2933-44.

21. Nagata N, Yasunaga H, Matsui H, Fushimi K, Watanabe K, Akiyama J, et al. Therapeutic endoscopy-related GI bleeding and thromboembolic events in patients using warfarin or direct oral anticoagulants: results from a large nationwide database analysis. Gut. 2018;67:1805-12.

22. Radaelli F, Fuccio L, Paggi S, Hassan C, Repici A, Rondonotti E, On behalf of Bowell Group, et al. Periendoscopic management of direct oral anticoagulants: a prospective cohort study. Gut. 2019;68:969-76.

23. Abe S, Oda I, Mori G, Nonaka S, Suzuki H, Yoshianaga S, et al. Complete endoscopic closure of a large gastric defect with endoloop and endoclips after complex endoscopic submucosal dissection. Endoscopy. 2015;47(Suppl 1):UCTN:E374-5.

24. Tsuji Y, Fujishiro M, Kodashima S, Ono S, Niimi K, Mochizuki $\mathrm{S}$, et al. Polyglycolic acid sheets and fibrin glue decrease the risk of bleeding after endoscopic submucosal dissection of gastric neoplasms (with video). Gastrointest Endosc. 2015;81:906-12.

25. Vanassche T, Hirsh J, Eikelboom JW, Ginsberg JS. Organ-specific bleeding patterns of anticoagulant therapy: lessons from clinical trials. Thromb Haemost. 2014;112:918-23.

26. Blech S, Ebner T, Ludwig-Schwellinger E, Stangier J, Roth W. The metabolism and disposition of the oral direct thrombin inhibitor, dabigatran, in humans. Drug Metab Dispos. 2008;36:386-99.

27. Desai JC, Chatterjee P, Friedman K, Aisenberg J. Incidence and clinical presentation of gastrointestinal bleeding in atrial fibrillation patients taking direct oral anticoagulants. Am J Gastroenterol Suppl. 2016;3:13-21.

28. Otuki S, Izumi D, Suda M, Sato A, Hasegawa Y, Yagihara N, et al. Effects of direct oral anticoagulants at the peak phase, trough phase, and after vascular injury. J Am Coll Cardiol. 2018;71:102-4.
29. Graham DJ, Reichman ME, Wernecke M, Hsueh YH, Izem R, Southworth MR, et al. Stroke, bleeding, and mortality risks in elderly medicare beneficiaries treated with dabigatran or Rivaroxaban for nonvalvular atrial fibrillation. JAMA Intern Med. 2016;176:1662-71.

30. Calkins H, Willems S, Gerstenfeld EP, Verma A, Schilling R, Hohnloser SH, et al. Uninterrupted dabigatran versus warfarin for ablation in atrial fibrillation. N Engl J Med. 2017;376:1627-36.

31. Garcia DA, Regan S, Henault LE, Upadhyay A, Baker J, Othman $\mathrm{M}$, et al. Risk of thromboembolism with short-term interruption of warfarin therapy. Arch Intern Med. 2008;168:63-9.

32. Harada H, Suehiro S, Murakami D, Shimizu T, Nakahara R, Katsuyama $\mathrm{Y}$, et al. Continuous use of low-dose warfarin for gastric endoscopic submucosal dissection: a prospective study. Endosc Int Open. 2017;5:E348-53.

33. Blacker DJ, Wijdicks EF, McClelland RL. Stroke risk in anticoagulated patients with atrial fibrillation undergoing endoscopy. Neurology. 2003;61:964-8.

34. Kato M, Uedo N, Hokimoto S, Ieko M, Higuchi K, Murakami $\mathrm{K}$, et al. Guidelines for gastroenterological endoscopy in patients undergoing antithrombotic treatment: 2017 appendix on anticoagulants including direct oral anticoagulants (DOACs). Dig Endosc. 2018;30:433-40.

35. Acosta RD, Abraham NS, Chandrasekhara V, Chathadi KV, Early DS, Eloubeidi MA, et al. The management of antithrombotic agents for patients undergoing GI endoscopy. Gastrointest Endosc. 2016;83:3-16

36. Veitch AM, Vanbiervliet G, Gershlick AH, Boustiere C, Baglin TP, Smith LA, et al. Endoscopy in patients on antiplatelet or anticoagulant therapy, including direct oral anticoagulants: British Society of Gastroenterology (BSG) and European Society of Gastrointestinal Endoscopy (ESGE) guidelines. Gut. 2016;65:374-89.

37. Chan FKL, Goh KL, Reddy N, Fujimoto K, Ho KY, Hokimoto S, et al. Management of patients on antithrombotic agents undergoing emergency and elective endoscopy: joint Asian Pacific Association of Gastroenterology (APAGE) and Asian Pacific Society for Digestive Endoscopy (APSDE) practice guidelines. Gut. 2018;67(3):405-17.

38. Kataoka Y, Tsuji Y, Hirasawa K, Takimoto K, Wada T, Mochizuki $\mathrm{S}$, et al. Endoscopic tissue shielding to prevent bleeding after endoscopic submucosal dissection: a prospective multicenter randomized controlled trial. Endoscopy. 2019;51:619-27.

Publisher's Note Springer Nature remains neutral with regard to jurisdictional claims in published maps and institutional affiliations.

\section{Affiliations}

\section{Hideomi Tomida ${ }^{1,2} \cdot$ Toshiyuki Yoshio $^{3}$ (D) $\cdot$ Kimihiro Igarashi $^{4} \cdot$ Yoshinori Morita $^{5} \cdot$ Ichiro Oda $^{6} \cdot$ Takuya Inoue $^{7}$. Takuto Hikichi $^{8}$. Tetsuya Sumiyoshi $^{9} \cdot$ Hisashi Doyama $^{10}$. Yosuke Tsuji ${ }^{11}$. Jun Nishikawa ${ }^{12}$. Waku Hatta ${ }^{13}$. Tatsuya Mikami ${ }^{14} \cdot$ Mikitaka Iguchi $^{15} \cdot$ Kazuki Sumiyama $^{16} \cdot$ Katsumi Yamamoto $^{17} \cdot$ Kazuya Kitamura $^{18}$. Shiko Kuribayashi ${ }^{19}$. Atsushi Yanagitani ${ }^{20}$. Toshio Uraoka ${ }^{21} \cdot$ Tomoyuki Yada $^{22} \cdot$ Kenkei Hasatani $^{23}$. Koichiro Kawaguchi ${ }^{24}$. Tomoki Fujita ${ }^{25}$. Tsutomu Nishida ${ }^{26}$ - Yoichi Hiasa ${ }^{1}$ - Mitsuhiro Fujishiro ${ }^{27}$ - for the FIGHT- Japan Study Group}

1 Department of Gastroenterology and Metabology, Ehime University Graduate School of Medicine, 454 Shitsukawa, Toon 7910295, Japan
2 Gastroenterology Center, Ehime Prefectural Central Hospital, Kasugamachi 83, Matsuyama 7900024, Japan 
3 Department of Gastroenterology, Cancer Institute Hospital, Japanese Foundation for Cancer Research, 3-8-31 Ariake, Koto-ku, Tokyo 1358550, Japan

4 Division of Endoscopy, Shizuoka Cancer Center, 1007 Shimonagakubo, Nagaizumi-cho, Sunto-gun, Shizuoka 4118777, Japan

5 Department of Gastroenterology, Kobe University Graduate School of Medicine, 7-5-1 Kusunoki-cho, Chuo-ku, Kobe 6500017, Japan

6 Endoscopy Division, National Cancer Center Hospital, 5-1-1 Tsukiji, Chuo-ku, Tokyo 1040045, Japan

7 Division of Gastroenterology and Hepatology, Osaka General Medical Center, 3-1-56 Bandai-higashi, Sumiyoshi-ku, Osaka 5588558, Japan

8 Department of Endoscopy, Fukushima Medical University Hospital, 1 Hikarigaoka, Fukushima 9601295, Japan

9 Department of Gastroenterology, Tonan Hospital, 3-8 Kita4-Nishi7, Chuo-ku, Sapporo 0600004, Japan

10 Department of Gastroenterology, Ishikawa Prefectural Central Hospital, 2-1 Kuratsuki-higashi, Kanazawa 9208530, Japan

11 Department of Gastroenterology, Graduate School of Medicine, The University of Tokyo, 7-3-1 Hongo, Bunkyo-ku, Tokyo 1138655, Japan

12 Faculty of Laboratory Science, Yamaguchi University Graduate School of Medicine, 1-1-1 Minamikogushi, Ube 7558505, Japan

13 Division of Gastroenterology, Tohoku University Graduate School of Medicine, 1-1 Seiryo-machi, Aoba-ku, Sendai 9808574, Japan

14 Division of Endoscopy, Hirosaki University Hospital, 53 Honcho, Hirosaki 0368563, Japan
15 Second Department of Internal Medicine, Wakayama Medical University, 811-1 Kimiidera, Wakayama 6410012, Japan

16 Department of Endoscopy, The Jikei University School of Medicine, 3-25-8 Nishi-shinbashi, Minato-ku, Tokyo 1058461, Japan

17 Department of Gastroenterology, JCHO Osaka Hospital, 4-2-78 Fukushima, Fukushima-ku, Osaka 5530003, Japan

18 Department of Gastroenterology, Kanazawa University Hospital, 13-1 Takara-machi, Kanazawa 9208641, Japan

19 Department of Gastroenterology and Hepatology, Gunma University Graduate School of Medicine, 3-39-15 Showa-machi, Maebashi 3718511, Japan

20 Department of Gastroenterology, Tottori Prefectural Central Hospital, 730 Ezu, Tottori 6800901, Japan

21 Department of Gastroenterology, National Hospital Organization Tokyo Medical Center, 2-5-1 Higashigaoka, Meguro-ku, Tokyo 1528902, Japan

22 Division of Gastroenterology and Hepatology, Kohnodai Hospital, National Center for Global Health and Medicine, 1-7-1 Kohnodai, Ichikawa 2728516, Japan

23 Department of Gastroenterology, Fukui Prefectural Hospital, 2-8-1 Yotsui, Fukui 9108526, Japan

24 Department of Gastroenterology, Tottori University School of Medicine, 36-1 Nishi-cho, Yonago 6838504, Japan

25 Department of Gastroenterology, Otaru Ekisaikai Hospital, 1-4-1 Inaho, Otaru 0470032, Japan

26 Department of Gastroenterology, Toyonaka Municipal Hospital, 4-14-1 Shibahara, Toyonaka 5608565, Japan

27 Department of Gastroenterology and Hepatology, Nagoya University Graduate School of Medicine, 65 Tsurumai-cho, Showa-ku, Nagoya 466-8550, Japan 